\title{
Pinning synchronization of nonlinearly coupled complex networks with time-varying delays using M-matrix strategies
}

\author{
Jingyi Wang ${ }^{\mathrm{a}}$, Jianwen Feng ${ }^{\mathrm{b}, *}$, Chen $\mathrm{Xu}^{\mathrm{b}, *}$, Yi Zhao ${ }^{\mathrm{b}}$, Jiqiang Feng ${ }^{\mathrm{b}}$ \\ ${ }^{a}$ College of Information and Engineering, Shenzhen University, Shenzhen 518060, PR China \\ ${ }^{b}$ College of Mathematics and Computational Science, Shenzhen University, Shenzhen 518060, PR China
}

\begin{abstract}
In this paper, we investigate the global synchronization problem for a class of nonlinearly-coupled complex networks (NCCNs) with mixed time-varying delays by using an M-matrix approach. The M-matrix strategy allows many synchronization problems with much relaxed conditions to be considered and can be used to derive sufficient conditions for this new class of NCCNs by pinning control. We also propose an effective way of adapting the coupling strengths of complex networks and give numerical examples to illustrate the effectiveness of our theoretical results.
\end{abstract}

Keywords: nonlinearly-coupled complex networks, synchronization, M-matrix, time-varying delays, pinning control.

\section{Introduction}

A complex network is a structure that is made up of a large set of nodes that are connected by a set of edges and they are ubiquitously found in nature and the modern world (for example, the Internet, mobile sensor networks, food networks and human communication networks). Complex networks have been extensively studied in the physical sciences and mathematics in the past few decades and much is already known about their dynamical behavior (see $[1,2,3,4,5,6,7]$ and the references therein). Synchronization (first discovered by Pecora and Carroll in 1990) is a process in which two or more dynamical systems seek to adjust a certain property of their motion to a common behavior in the limit as time tends to infinity [8]. Synchronized complex networks have wide-ranging applications to many fields (such as population dynamics, power systems and automatic control $[8,9,10]$ ) and many synchronization patterns have been discovered in the past (for example, complete synchronization [11, 12], cluster synchronization [13], phase synchronization [14] and so on). Techniques are also available that facilitate the attainment of synchronization such as by adaptive control $[15,16]$, intermittent control [17], impulsive control [18] and pinning control [19, 20, 21, 22].

As everyone knows, the real-world networks normally have a large number of nodes, and it is usually impractical to control a complex network by adding the controllers to all nodes [17]. Pinning control is a particularly useful technique that applies controllers to only a small fraction of the nodes in a network (thus greatly reducing the number of controlled nodes in real-world complex networks). In fact, pinning control is so effective in some networks that only one controller is required for synchronization [19]. And the pinning scheme of the most highly connected nodes induced that a significant reduction in required controllers as compared to the traditional control scheme [9]. In general, the exact number of nodes that are required to be pinned for any network and coupling strength is also completely known [23, 24].

Many networks in nature are nonlinearly-coupled (for example, neural and metabolic networks, in which the coupling configurations are continuously oscillating between two fixed states [25]). However, most synchronization studies that have been conducted so far have been confined to the smaller and less applicable class of complex networks that are linearly coupled [19, 20,23] thus leaving the more important and naturally occurring nonlinearly-coupled ones virtually uninvestigated. This paper attempts to fill this gap in our knowledge by investigating the nonlinearly coupled problem via pinning control using the M-matrix approach.

Pinning control is a very effective scheme for synchronization in complex networks by controlling only a small number of the system nodes [26]. The M-matrix is one of the most common ways of expressing the structure of a problem in the biological, physical and social sciences and it is also commonly used in mathematics to

\footnotetext{
* Corresponding author

Email addresses: fengjw@szu.edu.cn (Jianwen Feng), xuchen_szu@szu.edu.cn (Chen Xu)
} 
establish eigenvalue bounds. Song et al recently proposed an M-matrix method to investigate the first-order leader-follower synchronization problem in pinning-controlled multiagent systems [27] and the leader-follower consensus problem for multi-agent systems with inherent nonlinear dynamics [28]. Consensus Tracking of multi-agent systems with Lipschitz-type node dynamics were studied by using tools M-matrix theory in [29]. The M-matrix provides a more general framework for the formulation and solution of nonlinearly-coupled systems and allows for the derivation of sufficient conditions for synchronization.

The main aim of this paper is to extend the results of [27] to the more general problem of synchronizing nonlinearly coupled complex networks. In particular, we study the synchronization of nonlinearly-coupled complex networks with time-varying delays by using the pinning control method using the M-matrix approach. We also rigorously construct an adaptive feedback controller that can be used to completely synchronize any real-world network. Our results will be applicable to many synchronization problems in different fields of science and technology.

This paper is organized as follows. In Sect. 2, we state some preliminary definitions and lemmas that will be needed for the rest of the paper. In Sect. 3, we introduce the general model for a nonlinearly coupled complex network with time-varying delayed dynamical nodes and establish some synchronization criteria for these complex dynamical networks. In Sect. 4, we discuss some numerical examples of the theoretical results. Sect. 5 is the conclusion of the paper.

\section{Preliminaries}

\subsection{Notations}

Throughout this paper, $\mathbb{R}^{n}$ shall denote the $n$-dimensional Euclidean space and $\mathbb{R}^{n \times n}$ the set of all $n \times n$ real matrices. For a real matrix $A$, let $A^{\top}$ be its transpose and $A^{s}=\left(A+A^{\top}\right) / 2$ be its symmetric part. Let $\mathbf{1}_{n}=(1,1, \ldots, 1)^{\top} \in \mathbb{R}^{n}$ and $I_{n}$ be the $n$-dimensional identity matrix. For symmetric matrices $A$, the notation $A>0$ (respectively, $<0$ ) shall mean that $A$ is a positive-definite (respectively, negative-definite) matrix and $\lambda_{\max }(A)$ and $\lambda_{\min }(A)$ shall denote the greatest and least eigenvalues of a symmetric matrix respectively. For a square matrix $A \in \mathbb{C}^{n \times n}$, let $\lambda_{i}(A)$ be the $i$ th eigenvalue, $\rho(A)=\max _{1 \leq i \leq n}\left|\lambda_{i}(A)\right|$ be the spectral radius and $\mathcal{J}(A)=\min _{1 \leq i \leq n} \operatorname{Re}\left(\lambda_{i}(A)\right)$ be the minimum of the real parts of all the eigenvalues. The symbol $\otimes$ denotes the Kronecker product.

\subsection{Graph Theory and Matrix Algebra}

Let $\mathcal{G}=(\mathcal{V}, \mathcal{E}, \mathcal{A})$ be a weighted directed graph composed of a set of nodes $\mathcal{V}=\left\{v_{1}, \ldots, v_{n}\right\}$, a set of edges $\mathcal{E}=\mathcal{V} \times \mathcal{V}$ and a weighted adjacency matrix $\mathcal{A}=\left[a_{i j}\right]$ with non-negative adjacency elements $a_{i j}$. An edge of $\mathcal{G}$ is denoted by $e_{i j}=\left(v_{i}, v_{j}\right) \in \mathcal{E}$, which means that node $v_{j}$ receives information from node $v_{i}\left(v_{i}\right.$ is called the parent of $v_{j}$ ).The adjacency elements associated with the edges of the graph are positive, i.e., $e_{i j} \in \mathcal{E} \Leftrightarrow a_{i j}>0$. Moreover, we assume that $a_{i i}=0$ for all $i \in 1, \ldots, n$. The set of neighbors of node $v_{i}$ is denoted by $\mathcal{N}_{i}=\left\{v_{j} \in V:\left(v_{i}, v_{j}\right) \in \mathcal{E}\right\}$. The in-degree and out-degree of node $v_{i}$ are, respectively, defined as follows:

$$
\operatorname{deg}_{\text {in }}\left(v_{i}\right)=\sum_{j=1}^{n} a_{j i}, \quad \operatorname{deg}_{\text {out }}\left(v_{i}\right)=\sum_{j=1}^{n} a_{i j} .
$$

The degree matrix of digraph $\mathcal{G}$ is a diagonal matrix $\Delta=\left[\Delta_{i j}\right]$ where $\Delta_{i j}=0$ for all $i \neq j$, and $\Delta_{i i}=d_{e g_{\text {out }}}\left(v_{i}\right)$. The graph Laplacian associated with the digraph $\mathcal{G}$ is defined as

$$
\mathcal{L}(\mathcal{G})=L=\Delta-A
$$

A reversal of a graph is obtained by reversing the orientation of all the edges. If a graph has adjacency matrix $A$, then its reversal has adjacency matrix $A^{\top}$.

Firstly, some results on $M$-matrix are provided to study the pinning control synchronization of NCCNS.

Lemma 1. [27] The Laplacian matrix $L$ has a simple zero eigenvalue and all the other eigenvalues have positive real parts if and only if the diagraph associated with $L$ has a directed spanning tree.

Lemma 2. [27] Let $L$ be the Laplacian matrix of a diagraph $\mathcal{G}$. The minimum number of directed trees in $\mathcal{G}$ is equal to the multiplicity of the zero eigenvalue of $L$.

Definition 1. [30] The set $Z_{n} \in \mathbb{R}^{n \times n}$ is defined by

$$
Z_{n} \equiv\left\{A=\left[a_{i j}\right] \in \mathbb{R}^{n \times n}: a_{i j} \leq 0, i \neq j, \quad i, j=1,2, \ldots, n\right\}
$$


Definition 2. [30] A matrix $A \in \mathbb{R}^{n \times n}$ is said to be positive stable if $i_{+}(A)=n$, where $i_{+}(A)$ is the number of eigenvalues of $A$, counting multiplicities, with positive real part.

Definition 3. [30] A matrix $A$ is called an $M$-matrix if $A \in Z_{n}$ and $A$ is positive stable.

Lemma 3. [30] If $A \in Z_{n}$, the following statements are equivalent:

1) $A$ is positive stable, that is, $A$ is an $M$-matrix.

2) $A=\alpha I-P, P \geq 0, \alpha>\rho(P)$.

3) Every real eigenvalues of $A$ is positive.

4) There is a positive diagonal matrix $D$ such that $D A+A^{\top} D$ is positive definite; that is, there is a positive diagonal dominant.

Before proceeding further, the following essential lemmas are presented, which are used in proving the main result of this paper.

Lemma 4. [25] Let $\mathbf{1}_{n}=(1,1, \ldots, 1)^{\top}, I_{n}=\operatorname{diag}\{1,1, \ldots, 1\} \in \mathbb{R}^{n}$ and $Q=\left(q_{i j}\right)=I_{n}-\frac{1}{N} \mathbf{1}_{n} \cdot \mathbf{1}_{n}^{\top}$. For all zero-row-sum matrices $M \in \mathbb{R}^{m \times n}$ and $\theta>0$, we have

$$
x^{\top} M y=x^{\top} M Q y \leq \frac{1}{2}\left(\frac{1}{\theta} x^{\top} M M^{\top} x+\theta y^{\top} Q y\right) .
$$

Lemma 5. [31] If $Q \in \mathbb{R}^{n \times n}$ is such that $q_{i j}=q_{j i}$ and $q_{i i}=-\sum_{j=1, i \neq j}^{n} q_{i j}, i, j=1,2, \ldots, n$, then

$$
u^{\top} Q \otimes P v=\sum_{i=1}^{n} \sum_{j=1}^{n} u_{i} q_{i j} P v_{j}=-\sum_{j>i} q_{i j}\left(u_{i}-u_{j}\right) P\left(v_{i}-v_{j}\right)
$$

for all matrices $P \in \mathbb{R}^{m \times m}$ and vectors $u=\left(u_{1}^{\top}, u_{2}^{\top}, \ldots, u_{n}^{\top}\right)^{\top}$ and $v=\left(v_{1}^{\top}, v_{2}^{\top}, \ldots, v_{n}^{\top}\right)^{\top}$, where $u_{i}=$ $\left(u_{i 1}, u_{i 2}, \ldots, u_{i m}\right)^{\top}$ and $v_{i}=\left(v_{i 1}, v_{i 2}, \ldots, v_{i m}\right)^{\top}$.

Lemma 6. [30] Let $A \in \mathbb{R}^{n \times n}$ and $B \in \mathbb{R}^{m \times m}$. If $\lambda(A)$ is an eigenvalue of $A$ and $\lambda(B)$ is an eigenvalue of $B$, then $\lambda(A) \lambda(B)$ is an eigenvalue of $A \otimes B$.

Lemma 7. For matrices $A, B, C$ and $D$ with appropriate dimensions, one has

1) $(A \otimes B)^{\top}=A^{\top} \otimes B^{\top}$

2) $(A+B) \otimes C=A \otimes C+B \otimes C$

3) $(A \otimes B)(C \otimes D)=(A C) \otimes(B D)$.

\subsection{Model description}

Consider a complex network consisting of $N$ identical nodes with nondelayed and time-varying delayed nonlinear coupling

$$
\begin{aligned}
\dot{x}_{i}(t)= & f\left(t, x_{i}(t), x_{i}\left(t-\tau_{1}(t)\right)\right)+\sum_{j=1, i \neq j}^{N} c_{1} a_{i j} \Gamma\left[g\left(x_{j}(t)\right)-g\left(x_{i}(t)\right)\right] \\
& +\sum_{j=1, i \neq j}^{N} c_{2} b_{i j} \Gamma\left[h\left(x_{j}\left(t-\tau_{2}(t)\right)\right)-h\left(x_{i}\left(t-\tau_{2}(t)\right)\right)\right], \quad i=1,2, \ldots, N,
\end{aligned}
$$

where $x_{i}(t)=\left(x_{i 1}(t), x_{i 2}(t), \ldots, x_{i n}(t)\right)^{\top} \in \mathbb{R}^{n}$ is the state vector of the $i$ th node of the network, $f\left(t, x_{i}(t), x_{i}(t-\right.$ $\left.\left.\tau_{1}(t)\right)\right)=\left[f_{1}\left(t, x_{i}(t), x_{i}\left(t-\tau_{1}(t)\right)\right), f_{2}\left(t, x_{i}(t), x_{i}\left(t-\tau_{1}(t)\right)\right), \ldots, f_{n}\left(t, x_{i}(t), x_{i}\left(t-\tau_{1}(t)\right)\right)\right]^{\top}$ is a continuous vectorvalued function, $\Gamma=\operatorname{diag}\left(\gamma_{1}, \gamma_{2}, \ldots, \gamma_{n}\right)$ is an inner coupling of the network that satisfies $\gamma_{j}>0, j=1,2, \ldots, n$, $A=\left[a_{i j}\right] \in \mathbb{R}^{n \times n}$ and $B=\left[b_{i j}\right] \in \mathbb{R}^{n \times n}$ are the outer coupling matrices of the network at time $t$ and $t-\tau_{2}(t)$ respectively such that $a_{i j} \geq 0$ for $i \neq j, a_{i i}=-\sum_{j=1, j \neq i}^{N} a_{i j}, b_{i j} \geq 0$ for $i \neq j$ and $b_{i i}=-\sum_{j=1, j \neq i}^{N} b_{i j}, \tau_{1}(t)$ is the inner time-varying delay, $\tau_{2}(t)$ is the coupling time-varying delay and the nonlinear coupling functions $h(\cdot)$ : $\mathbb{R}^{n} \rightarrow \mathbb{R}^{n}$ and $g(\cdot): \mathbb{R}^{n} \rightarrow \mathbb{R}^{n}$ are continuous and of the form $g\left(x_{i}(t)\right)=\left(g_{1}\left(x_{i 1}(t)\right), g_{2}\left(x_{i 2}(t)\right), \ldots, g_{n}\left(x_{i n}(t)\right)\right)^{\top}$ and $h\left(x_{i}(t)\right)=\left(h_{1}\left(x_{i 1}\left(t-\tau_{2}(t)\right)\right), h_{2}\left(x_{i 2}\left(t-\tau_{2}(t)\right)\right), \ldots, h_{n}\left(x_{i n}\left(t-\tau_{2}(t)\right)\right)\right)^{\top}$.

The initial conditions associated with (1) are

$$
x_{i}(s)=\varphi_{i}(s), \quad-\check{\tau} \leq s \leq 0, \quad i=1,2, \ldots, N
$$


where $\check{\tau}=\max \left\{\tau_{1}(t), \tau_{2}(t)\right\}$ and $\varphi_{i} \in \mathcal{C}\left([-\check{\tau}, 0], \mathbb{R}^{n}\right)$ with the norm $\left\|\varphi_{i}\right\|^{2}=\sup _{-\check{\tau} \leq s \leq 0} \varphi_{i}(s)^{\top} \varphi_{i}(s)$ and our objective is to control system (1) so that it stays in the trajectory $s(t) \in \mathbb{R}^{n}$ of the system

$$
\dot{s}(t)=f\left(t, s(t), s\left(t-\tau_{1}(t)\right)\right)
$$

by adding pinning controllers to some of the nodes. Without loss of generality, let the first $l$ nodes be controlled. Then the network is described by

$$
\begin{aligned}
\dot{x}_{i}(t)= & f\left(t, x_{i}(t), x_{i}\left(t-\tau_{1}(t)\right)\right)+\sum_{j=1, i \neq j}^{N} c_{1} a_{i j} \Gamma\left(g\left(x_{j}(t)\right)-g\left(x_{i}(t)\right)\right) \\
& +\sum_{j=1, i \neq j}^{N} c_{2} b_{i j} \Gamma\left(h\left(x_{j}\left(t-\tau_{2}(t)\right)\right)-h\left(x_{i}\left(t-\tau_{2}(t)\right)\right)\right)+u_{i}(t), \quad i=1,2, \ldots, N,
\end{aligned}
$$

where $u_{i}(t)(i=1,2, \ldots, N)$ are the linear state feedback controllers that are defined by

$$
u_{i}(t)= \begin{cases}-c_{1} \varepsilon_{i} \Gamma\left(x_{i}(t)-s(t)\right), & i=1,2, \ldots, l \\ 0, & i=l+1, l+2, \ldots, N\end{cases}
$$

for some control gains $\varepsilon_{i}>0(i=1,2, \ldots, l)$, denoted by $\Xi=\operatorname{diag}\left\{\varepsilon_{1}, \varepsilon_{2}, \ldots, \varepsilon_{l}, 0, \ldots, 0\right\} \in \mathbb{R}^{n \times n}$. Define $e_{i}(t)=x_{i}(t)-s(t)(i=1,2, \ldots, N)$ as the synchronization error. Then, according to the controller (4), the error system is

$$
\begin{aligned}
\dot{e}_{i}(t)= & f\left(t, x_{i}(t), x_{i}\left(t-\tau_{1}(t)\right)\right)-f\left(t, s_{i}(t), s_{i}\left(t-\tau_{1}(t)\right)\right)+\sum_{j=1}^{N} a_{i j} \Gamma\left(h\left(x_{j}(t)\right)-h(s(t))\right) \\
& +\sum_{j=1}^{N} b_{i j} \Gamma\left(g\left(x_{j}\left(t-\tau_{2}(t)\right)\right)-g\left(s\left(t-\tau_{2}(t)\right)\right)\right)+u_{i}(t), \quad i=1,2, \ldots, N .
\end{aligned}
$$

Definition 4. The complex network (3) is said to be synchronized if the trivial solution of system (5) is such that

$$
\sum_{i=1}^{N} \lim _{t \rightarrow \infty}\left\|e_{i}\left(t, t_{0}, \varphi_{i}\right)\right\|=0
$$

for any initial data $\varphi_{i} \in \mathcal{C}\left([-\tau, 0] ; \mathbb{R}^{n}\right)$.

Now, we introduce the following assumptions to study the synchronization of network (1).

Assumption 1. [17] For each function $f(\cdot)$ in network (1), there exists a positive definite diagonal matrix $P=\operatorname{diag}\left\{p_{1}, p_{2}, \ldots, p_{n}\right\}$, a diagonal matrix $\Delta=\operatorname{diag}\left\{\delta_{1}, \delta_{2}, \ldots, \delta_{n}\right\}$ and constants $\eta>0, \theta>0$ such that

$$
(x-y)^{\top} P((f(t, x, z)-f(t, y, w))-\Delta \Gamma(x-y)) \leq-\eta(x-y)^{\top}(x-y)+\zeta(z-w)^{\top}(z-w)
$$

for some given matrix $\Gamma$ and all $x, y, z, w \in \mathbb{R}^{n}$.

Remark 1. A continuous function $f$ is said to belong to the function class QUAD, denoted by $f \in \operatorname{QUAD}(P, \Delta, \eta, \zeta)$, if $f(\cdot)$ satisfies Assumption 1. One can easily verify that the function class QUAD includes almost all the chaotic systems such as the Lorenz system, the Rössler system, the Chen system, the delayed Chua's circuit, the logistic delayed differential system, the delayed Hopfield neural network and the delayed CNNs.

Assumption 2. [25] For each nonlinear coupling function $h_{i}(\cdot)$ and $g_{i}(\cdot)$ in network (1), there exist two nonnegative scalers $\beta_{i}^{h}, \varepsilon_{i}^{h}, \beta_{i}^{g}$ and $\varepsilon_{i}^{g}$ such that $h_{i}(x)-\beta_{i}^{h} x$ and $g_{i}(x)-\beta_{i}^{g} x$ satisfies the Lipschitz condition

$$
\left|h_{i}\left(x_{1}\right)-h_{i}\left(x_{2}\right)-\beta_{i}^{h}\left(x_{1}-x_{2}\right)\right| \leq \varepsilon_{i}^{h}\left|x_{1}-x_{2}\right|
$$

and

$$
\left|g_{i}\left(x_{1}\right)-g_{i}\left(x_{2}\right)-\beta_{i}^{g}\left(x_{1}-x_{2}\right)\right| \leq \varepsilon_{i}^{g}\left|x_{1}-x_{2}\right|,
$$

respectively, for all $x_{1}, x_{2} \in R, i=1,2, \ldots, n$. 


\section{Main Results}

In the section, we derive a sufficient condition for the attainment of synchronization by pinning control with the M-matrix approach.

Theorem 1. Under assumption 1 and 2 , if $\tau_{1}(t)$ and $\tau_{2}(t)$ are bounded and continuously differentiable functions such that $\dot{\tau}_{1}(t)<\bar{\tau}_{1}<1$ and $\dot{\tau}_{2}(t)<\bar{\tau}_{2}<1$ and

$$
\mathcal{J}\left(-c_{1}\left(A \otimes \Upsilon_{g}^{\beta} P \Gamma-\Xi \otimes P \Gamma\right)\right)>\rho,
$$

where $\rho=\lambda_{\max }\left(-\eta \Psi \otimes I_{n}+\Psi \otimes P \Delta \Gamma+c_{1} \Psi A A^{\top} \Psi \otimes P \Gamma \Theta_{g}^{-1}+2\left(1-\frac{1}{N}\right) I_{N} \otimes\left(\Upsilon_{g}^{\varepsilon}\right)^{2} P \Gamma \Theta_{g}+c_{2} \Psi B B^{\top} \Psi \otimes P \Gamma \Theta_{h}^{-1}+\right.$ $\Psi \otimes Q+\Psi \otimes R), \Upsilon_{g}^{\varepsilon}=\operatorname{diag}\left\{\varepsilon_{1}^{g}, \ldots, \varepsilon_{n}^{g}\right\}, \Upsilon_{g}^{\beta}=\operatorname{diag}\left\{\beta_{1}^{g}, \ldots, \beta_{n}^{g}\right\}, \Upsilon_{h}^{\varepsilon}=\operatorname{diag}\left\{\varepsilon_{1}^{h}, \ldots, \varepsilon_{n}^{h}\right\}, \Upsilon_{h}^{\beta}=\operatorname{diag}\left\{\beta_{1}^{h}, \ldots, \beta_{n}^{h}\right\}$, $Q=\frac{\zeta}{1-\bar{\tau}_{1}} I_{n}+\nu_{1} I_{n}, R=2 c_{2} \lambda_{\max }(\Psi) \frac{N-1}{N\left(1-\bar{\tau}_{2}\right)}\left(\Upsilon_{h}^{\varepsilon}+\Upsilon_{h}^{\beta}\right)^{2} P \Gamma \Theta_{h}+\nu_{2} I_{n}, \Theta_{h}, \Theta_{g}$ are positive definite matrices, $\Psi$ is a positive definite diagonal matrix and $\nu_{1}$ and $\nu_{2}$ are positive scalars, then the pinning-controlled complex network (3) globally asymptotically synchronizes to the isolated node (2).

Proof. Let $\lambda_{i}$ be an eigenvalue of $-c_{1} A \otimes \Upsilon_{g}^{\beta} P \Gamma-\Xi \otimes P \Gamma$. Then the existence of a directed spanning tree in $\mathcal{G}$ ensures that $R\left(\lambda_{i}\right)>0$ and the pinning condition (7) implies that $-c_{1} A \otimes \Upsilon_{g}^{\beta} P \Gamma-\Xi \otimes P \Gamma$ is an M-matrix. Hence, there exists a positive definite diagonal matrix $\Psi=\operatorname{diag}\left\{\xi_{1}, \ldots, \xi_{N}\right\}$ such that

$$
\left[\Psi\left(c_{1}\left(A \otimes \Upsilon_{g}^{\beta} P \Gamma-\Xi \otimes P \Gamma\right)-\rho I_{n N}\right)\right]^{S}<0 .
$$

Choose the Lyapunov-Krasovskii function as

$$
V(t, e(t))=\frac{1}{2} e(t)^{\top} \Psi \otimes P e(t)+\int_{t-\tau_{1}(t)}^{t} e(s)^{\top} \Psi \otimes Q e(s) d s+\int_{t-\tau_{2}(t)}^{t} e(s)^{\top} \Psi \otimes R e(s) d s,
$$

where $e(t)=\left(e_{1}(t)^{\top}, e_{2}(t)^{\top}, \ldots, e_{N}(t)^{\top}\right)^{\top}$ and let $e^{k}(t)=\left(e_{1 k}(t), e_{2 k}(t), \ldots, e_{N k}(t)\right)^{\top}, \tilde{g}^{k}\left(x^{k}(t)\right)=\left(g_{k}\left(x_{1 k}(t)\right)\right.$, $\left.g_{k}\left(x_{2 k}(t)\right), \ldots, g_{k}\left(x_{N k}(t)\right)\right)^{\top}, \tilde{\epsilon}_{g}^{k}\left(x^{k}(t)\right)=\left(\epsilon_{g, k}\left(x_{1 k}(t)\right), \epsilon_{g, k}\left(x_{2 k}(t)\right), \ldots, \epsilon_{g, k}\left(x_{N k}(t)\right)\right)^{\top}(k=1,2, \ldots, n)$. Then differentiate $V(t)$ along system (5) to get

$$
\begin{aligned}
\dot{V}(t, e(t))= & \sum_{i=1}^{N} \xi_{i} e_{i}(t)^{\top} P\left\{f\left(t, x_{i}(t), x_{i}\left(t-\tau_{1}(t)\right)\right)-f\left(t, s(t), s\left(t-\tau_{1}(t)\right)\right)+\sum_{j=1}^{N} c_{1} a_{i j} \Gamma g\left(x_{j}(t)\right)\right. \\
& \left.+\sum_{j=1}^{N} c_{2} b_{i j} \Gamma h\left(x_{j}\left(t-\tau_{2}(t)\right)\right)-c_{1} \varepsilon_{i} \Gamma e_{i}(t)\right\}+e(t)^{\top} \Psi \otimes Q e(t)-\left(1-\dot{\tau}_{1}\right) e\left(t-\tau_{1}(t)\right)^{\top} \Psi \otimes Q e\left(t-\tau_{1}(t)\right) \\
& +e(t)^{\top} \Psi \otimes R e(t)-\left(1-\dot{\tau}_{2}\right) e\left(t-\tau_{2}(t)\right)^{\top} \Psi \otimes R e\left(t-\tau_{2}(t)\right) \\
= & \sum_{i=1}^{N} \xi_{i} e_{i}(t)^{\top} P\left\{f\left(t, x_{i}(t), x_{i}\left(t-\tau_{1}(t)\right)\right)-f\left(t, s(t), s\left(t-\tau_{1}(t)\right)\right)-\Delta \Gamma e_{i}(t)\right\} \\
& +e(t)^{\top} \Psi \otimes P \Delta \Gamma e(t)+\sum_{i=1}^{N} \sum_{j=1}^{N} c_{1} \xi_{i} a_{i j} e_{i}(t)^{\top} P \Gamma g\left(x_{j}(t)\right)+\sum_{i=1}^{N} \sum_{j=1}^{N} c_{2} \xi_{i} b_{i j} e_{i}(t)^{\top} P \Gamma h\left(x_{j}\left(t-\tau_{2}(t)\right)\right) \\
& -c_{1} e(t)^{\top} \Psi \Xi \otimes P \Gamma e(t)+e(t)^{\top} \Psi \otimes Q e(t)-\left(1-\dot{\tau}_{1}\right) e\left(t-\tau_{1}(t)\right)^{\top} \Psi \otimes Q e\left(t-\tau_{1}(t)\right) \\
& +e(t)^{\top} \Psi \otimes R e(t)-\left(1-\dot{\tau}_{2}\right) e\left(t-\tau_{2}(t)\right)^{\top} \Psi \otimes \operatorname{Re}\left(t-\tau_{2}(t)\right) .
\end{aligned}
$$

As assumption 2, we have

$$
\begin{aligned}
& \sum_{i=1}^{N} \sum_{j=1}^{N} \xi_{i} e_{i}(t)^{\top} P a_{i j} \Gamma\left(g\left(x_{j}(t)\right)-g(s(t))\right) \\
= & \sum_{k=1}^{n} p_{k} \gamma_{k} e^{k}(t)^{\top} \Psi A\left[\tilde{g}^{k}\left(x^{k}(t)\right)-\tilde{g}^{k}\left(s^{k}(t)\right)\right] \\
= & \sum_{k=1}^{n} \beta_{k}^{g} p_{k} \gamma_{k} e^{k}(t)^{\top} \Psi A e^{k}(t)+\sum_{k=1}^{n} p_{k} \gamma_{k} e^{k}(t)^{\top} \Psi A\left[\tilde{\epsilon}_{g}^{k}\left(x^{k}(t)\right)-\tilde{\epsilon}_{g}^{k}\left(s^{k}(t)\right)\right] .
\end{aligned}
$$

Furthermore, since $A$ is a zero-row-sum matrix and assumption 2 (by Lemma 4 and Lemma 5), we have

$$
\sum_{k=1}^{n} p_{k} \gamma_{k} e^{k}(t)^{\top} \Psi A\left[\tilde{\epsilon}_{g}^{k}\left(x^{k}(t)\right)-\tilde{\epsilon}_{g}^{k}\left(s^{k}(t)\right)\right]
$$




$$
\begin{aligned}
\leq & \sum_{k=1}^{n} \frac{p_{k} \gamma_{k}}{\theta_{k}} e^{k}(t)^{\top} \Psi A A^{\top} \Psi e^{k}(t)+\sum_{k=1}^{n} p_{k} \gamma_{k} \theta_{k}\left[\tilde{\epsilon}_{k}\left(x^{k}(t)\right)-\tilde{\epsilon}_{k}\left(s^{k}(t)\right)\right]^{\top} Q\left[\tilde{\epsilon}_{k}\left(x^{k}(t)\right)-\tilde{\epsilon}_{k}\left(s^{k}(t)\right)\right] \\
\leq & \sum_{k=1}^{n} \frac{p_{k} \gamma_{k}}{\theta_{k}} e^{k}(t)^{\top} \Psi A A^{\top} \Psi e^{k}(t) \\
& -\sum_{k=1}^{n} p_{k} \gamma_{k} \theta_{k} \sum_{j>i} q_{i j}\left(\left(\gamma\left(x_{k j}(t)\right)-\gamma\left(s_{k}(t)\right)\right)-\left(\gamma\left(x_{k i}(t)\right)-\gamma\left(s_{k}(t)\right)\right)\right)^{2} \\
\leq & \sum_{k=1}^{n} \frac{p_{k} \gamma_{k}}{\theta_{k}} e^{k}(t)^{\top} \Psi A A^{\top} \Psi e^{k}(t) \\
& -2 \sum_{k=1}^{n} p_{k} \gamma_{k} \theta_{k} \sum_{j>i} q_{i j}\left(\left(\gamma\left(x_{k j}(t)\right)-\gamma\left(s_{k}(t)\right)\right)^{2}+\left(\gamma\left(x_{k i}(t)\right)-\gamma\left(s_{k}(t)\right)\right)^{2}\right) \\
\leq & \sum_{k=1}^{n} \frac{p_{k} \gamma_{k}}{\theta_{k}} e^{k}(t)^{\top} \Psi A A^{\top} \Psi e^{k}(t)-2 \varepsilon^{2} \sum_{k=1}^{n} p_{k} \gamma_{k} \theta_{k} \sum_{j>i} q_{i j}\left(e_{k j}(t)^{2}+e_{k i}(t)^{2}\right) \\
= & \sum_{k=1}^{n} \frac{p_{k} \gamma_{k}}{\theta_{k}} e^{k}(t)^{\top} \Psi A A^{\top} \Psi e^{k}(t)+2 \sum_{k=1}^{n} \varepsilon_{k}^{2} p_{k} \gamma_{k} \theta_{k}\left(1-\frac{1}{N}\right) e^{k}(t)^{\top} e^{k}(t) .
\end{aligned}
$$

It therefore follows from inequalities (9) and (10) that

$$
\begin{aligned}
& \sum_{i=1}^{N} \sum_{j=1}^{N} e_{i}(t)^{\top} P a_{i j} \Gamma\left(g\left(x_{j}(t)\right)-g(s(t))\right) \\
\leq & e(t)^{\top}\left(\Psi A \otimes \Upsilon_{g}^{\beta} P \Gamma+\Psi A A^{\top} \Psi \otimes P \Gamma \Theta_{g}^{-1}+2\left(1-\frac{1}{N}\right) I_{N} \otimes\left(\Upsilon_{g}^{\varepsilon}\right)^{2} P \Gamma \Theta_{g}\right) e(t) .
\end{aligned}
$$

Similarly, we have

$$
\begin{aligned}
& \sum_{i=1}^{N} \sum_{j=1}^{N} e_{i}(t)^{\top} P b_{i j} \Gamma\left(h\left(x_{j}\left(t-\tau_{2}(t)\right)\right)-h\left(s\left(t-\tau_{2}(t)\right)\right)\right) \\
\leq & \left.e(t)^{\top}\left(\Psi B B^{\top} \Psi \otimes P \Gamma \Theta_{h}^{-1}\right) e(t)+e\left(t-\tau_{2}(t)\right)^{\top} 2\left(1-\frac{1}{N}\right) I_{N} \otimes\left(\Upsilon_{h}^{\varepsilon}+\Upsilon_{h}^{\beta}\right)^{2} P \Gamma \Theta_{h}\right) e\left(t-\tau_{2}(t)\right)
\end{aligned}
$$

and after substituting inequalities (11-12) into equation (8), we have

$$
\begin{aligned}
\dot{V}(t) \leq & e(t)^{\top}\left(-\eta \Psi \otimes I_{n}+c_{1} \Psi A \otimes \Upsilon_{g}^{\beta} P \Gamma-c_{1} \Psi \Xi \otimes P \Gamma+\Psi \otimes P \Delta \Gamma+c_{1} \Psi A A^{\top} \Psi \otimes P \Gamma \Theta_{g}^{-1}\right. \\
& \left.+2\left(1-\frac{1}{N}\right) I_{N} \otimes\left(\Upsilon_{g}^{\varepsilon}\right)^{2} P \Gamma \Theta_{g}+c_{2} \Psi B B^{\top} \Psi \otimes P \Gamma \Theta_{h}^{-1}+\Psi \otimes Q+\Psi \otimes R\right) e(t) \\
+ & e\left(t-\tau_{1}(t)\right)^{\top}\left(\zeta \Psi \otimes I_{n}-\left(1-\bar{\tau}_{1}\right) \Psi \otimes Q\right) e\left(t-\tau_{1}(t)\right) \\
+ & e\left(t-\tau_{2}(t)\right)^{\top}\left(2 c_{2}\left(1-\frac{1}{N}\right) I_{N} \otimes\left(\Upsilon_{h}^{\varepsilon}+\Upsilon_{h}^{\beta}\right)^{2} P \Gamma \Theta_{h}-\left(1-\bar{\tau}_{2}\right) \Psi \otimes R\right) e\left(t-\tau_{2}(t)\right) .
\end{aligned}
$$

Let $y(t)=\left(e(t)^{\top}, e\left(t-\tau_{1}(t)\right)^{\top}, e\left(t-\tau_{2}(t)\right)^{\top}\right)^{\top}$ and recall that $Q=\frac{\zeta}{1-\bar{\tau}_{1}} I_{n}+\nu_{1} I_{n}$ and $R=2 c_{2} \lambda_{\max }(\Psi) \frac{N-1}{N\left(1-\bar{\tau}_{2}\right)}\left(\Upsilon_{h}^{\varepsilon}+\right.$ $\left.\Upsilon_{h}^{\beta}\right)^{2} P \Gamma \Theta_{h}+\nu_{2} I_{n}$. Then inequality (13) implies that $\dot{V}(t) \leq 0$ and $\dot{V}(t)=0$ if and only if $y(t)=0_{3 N n}$. So the set $M=\left\{y(t): y(t)=0_{3 N n}\right\}$ is the largest invariant set contained in the set $D=\{y(t) \mid V(t)=0\}$ for system (5) and thus every solution of system (5) (starting from any initial condition) approaches $M$ as $t \rightarrow \infty$ (according to LaSalle's invariance principle). Therefore $\left\|e_{i}(t)\right\| \rightarrow 0, i=1,2, \ldots, N$ and the pinning-controlled network (3) globally asymptotically synchronizes to the isolated node (1).

Remark 2. Even though the network (3) can be synchronized as long as the coupling strength satisfies $\mathcal{J}\left(-c_{1}\left(A \otimes \Upsilon_{g}^{\beta} P \Gamma-\Xi \otimes P \Gamma\right)\right)>\rho$ (see condition (7), the theoretical value required for the coupling strength is usually much larger than that needed in practice. We shall next use an adaptive approach to find a sharp bound for this coupling strength.

To make Theorem 1 more applicative, we give the following corollaries.

When $h_{i}(x)=x$ and $g_{i}(x)=x$ are linear functions, system (3) is a linearly-coupled system and we have the following result. 
Corollary 1. Under assumption 1 , if $\tau_{1}(t)$ and $\tau_{2}(t)$ are bounded and continuously differentiable functions such that $\dot{\tau}_{1}(t)<\bar{\tau}_{1}<1$ and $\dot{\tau}_{2}(t)<\bar{\tau}_{2}<1$ and

$$
\mathcal{J}\left(-c_{1}(A \otimes P \Gamma-\Xi \otimes P \Gamma)\right)>\rho,
$$

where $\rho=\lambda_{\max }\left(-\eta \Psi \otimes I_{n}+\Psi \otimes P \Delta \Gamma+c_{2} \Psi B B^{\top} \Psi \otimes P \Gamma \Theta_{h}^{-1}+\Psi \otimes Q+\Psi \otimes R\right), Q=\frac{\zeta}{1-\bar{\tau}_{1}} I_{n}+\nu_{1} I_{n}$, $R=2 c_{2} \lambda_{\max }(\Psi) \frac{N-1}{N\left(1-\bar{\tau}_{2}\right)} P \Gamma \Theta_{h}+\nu_{2} I_{n}, \Theta_{h}$ is a positive definite matrices, $\Psi$ is a positive definite diagonal matrix and $\nu_{1}$ and $\nu_{2}$ are positive scales, then the pinning-controlled complex network (3) globally asymptotically synchronizes to the isolated node (2).

When $h_{i}(x)=x$ is a linear function and $t_{1}(t)=\tau$ and $c_{2}=0$ (without time-delay coupling), then system (3) is a linearly-coupled system without time-delay coupling and we have the following result.

Corollary 2. Under assumption 1, if

$$
\mathcal{J}\left(-c_{1}(A \otimes P \Gamma-\Xi \otimes P \Gamma)\right)>\rho,
$$

where $\rho=\lambda_{\max }\left(-\eta \Psi \otimes I_{n}+\Psi \otimes P \Delta \Gamma+\Psi \otimes Q\right), Q=\zeta I_{n}+\nu_{1} I_{n}, \Theta_{h}$ is a positive definite matrices, $\Psi$ is a positive definite diagonal matrix and $\nu_{1}$ and $\nu_{2}$ are positive scalars, then the pinning-controlled complex network (3) globally asymptotically synchronizes to the isolated node (2)

Remark 3. Corollary 2 gives a sufficient condition for the synchronization of linearly coupled complex networks using pinning control that is the same as that obtained by Q. Song et. al. (Theorem 2 in Ref. [27]). We can therefore say that Theorem 1 is a nontrivial extension of the result obtained in [27].

Real-world complex networks have coupling strengths that are usually less than their theoretical values [19]. The following theorem, however, allows us to accommodate for relatively small coupling strengths by using an adaptive feedback control technique.

Let the coupling strengths of the network functions be time-varying (we shall now design the corresponding coupling strength adaptive laws). System (3) can be written as

$$
\begin{aligned}
\dot{x}_{i}(t)= & f\left(t, x_{i}(t), x_{i}\left(t-\tau_{1}(t)\right)\right)+\sum_{j=1, i \neq j}^{N} c_{1}(t) a_{i j} \Gamma\left(g\left(x_{j}(t)\right)-g\left(x_{i}(t)\right)\right) \\
& +\sum_{j=1, i \neq j}^{N} c_{2} b_{i j} \Gamma\left(h\left(x_{j}\left(t-\tau_{2}(t)\right)\right)-h\left(x_{i}\left(t-\tau_{2}(t)\right)\right)\right)+u_{i}(t) \quad i=1,2, \ldots, N,
\end{aligned}
$$

where $c_{1}(t)$ is an adaptive coupling strength that can be suitably chosen to synchronize system (14). Design a local feedback controller

$$
u_{i}(t)= \begin{cases}-c_{1}(t) \varepsilon_{i} \Gamma\left(x_{i}(t)-s(t)\right), & i=1,2, \ldots, l \\ 0, & i=l+1, l+2, \ldots, N\end{cases}
$$

Theorem 2. Under assumption 1 and 2, if $\tau_{1}(t)$ and $\tau_{2}(t)$ are bounded and continuously differentiable functions such that $\dot{\tau}_{1}(t)<\bar{\tau}_{1}<1$ and $\dot{\tau}_{2}(t)<\bar{\tau}_{2}<1$. Let $c_{1}(t)$ be an adaptive coupling strength such that

$$
\dot{c}_{1}(t)=\alpha \sum_{i=1}^{N} \xi_{i} e_{i}(t)^{\top} P e_{i}(t)=\alpha e(t)^{\top} \Psi \otimes P e(t)
$$

for some $\alpha>0$ and initial value $c_{1}(0) \geq 0$ and $\Psi$ is a positive definite diagonal matrix. Then system (14) can be synchronized.

Proof. Choose a constant $\alpha>0$ and let $e_{i}(t)=x_{i}(t)-s(t)$. Construct the following Lyapunov-Krasovskii function

$$
V(t)=\frac{1}{2} e(t)^{\top} \Psi \otimes P e(t)+\int_{t-\tau_{1}(t)}^{t} e(s)^{\top} \Psi \otimes Q e(s) d s+\int_{t-\tau_{2}(t)}^{t} e(s)^{\top} \Psi \otimes R e(s) d s+\frac{1}{2 \alpha \kappa}\left(c-\kappa c_{1}(t)\right)^{2}
$$

for some constants $c$ and $\kappa$ that will be determined later. Differentiating $V(t)$ gives

$$
\frac{d V(t)}{d t}=\sum_{i=1}^{N} \xi_{i} e_{i}(t)^{\top} P\left\{f\left(t, x_{i}(t), x_{i}\left(t-\tau_{1}(t)\right)\right)-f\left(t, s(t), s\left(t-\tau_{1}(t)\right)\right)+\sum_{j=1}^{N} c_{1}(t) a_{i j} \Gamma g\left(x_{j}(t)\right)\right.
$$




$$
\begin{aligned}
& \left.+\sum_{j=1}^{N} c_{2} b_{i j} \Gamma h\left(x_{j}\left(t-\tau_{2}(t)\right)\right)-c_{1} \varepsilon_{i} \Gamma e_{i}(t)\right\}+e(t)^{\top} \Psi \otimes Q e(t)-\left(1-\dot{\tau}_{1}\right) e\left(t-\tau_{1}(t)\right)^{\top} \Psi \otimes Q e\left(t-\tau_{1}(t)\right) \\
& +e(t)^{\top} \Psi \otimes \operatorname{Re}(t)-\left(1-\dot{\tau}_{2}\right) e\left(t-\tau_{2}(t)\right)^{\top} \Psi \otimes R e\left(t-\tau_{2}(t)\right)-c e(t)^{\top} \Psi \otimes P \Gamma e(t)+\kappa c_{1}(t) e(t)^{\top} \Psi \otimes P \Gamma e(t)
\end{aligned}
$$

and so, by Theorem 1 , we have

$$
\begin{aligned}
\dot{V}(t) \leq & e(t)^{\top}\left(c_{1}(t) \Psi A \otimes \Upsilon_{g}^{\beta} P \Gamma-c_{1}(t) \Psi \Xi \otimes P \Gamma+\kappa c_{1}(t) \Psi \otimes P \Gamma+c_{1}(t) \Psi A A^{\top} \Psi \otimes P \Gamma \Theta_{g}^{-1}+2\left(1-\frac{1}{N}\right) I_{N} \otimes\left(\Upsilon_{g}^{\varepsilon}\right)^{2} P \Gamma \Theta_{g}\right. \\
& \left.-\eta \Psi \otimes I_{n}-c \Psi \otimes P \Gamma+\Psi \otimes P \Delta \Gamma+c_{2} \Psi B B^{\top} \Psi \otimes P \Gamma \Theta_{h}^{-1}+\Psi \otimes Q+\Psi \otimes R\right) e(t) \\
& +e\left(t-\tau_{1}(t)\right)^{\top}\left(\zeta \Psi \otimes I_{n}-\left(1-\bar{\tau}_{1}\right) \Psi \otimes Q\right) e\left(t-\tau_{1}(t)\right) \\
& +e\left(t-\tau_{2}(t)\right)^{\top}\left(2 c_{2}\left(1-\frac{1}{N}\right) I_{N} \otimes\left(\Upsilon_{h}^{\varepsilon}+\Upsilon_{h}^{\beta}\right)^{2} P \Gamma \Theta_{h}-\left(1-\bar{\tau}_{2}\right) \Psi \otimes R\right) e\left(t-\tau_{2}(t)\right) .
\end{aligned}
$$

Hence, by choosing $\kappa$ and $c$ such that $\Psi A \otimes \Upsilon_{g}^{\beta} P \Gamma-\Psi \Xi \otimes P \Gamma+\kappa \Psi \otimes P \Gamma+\Psi A A^{\top} \Psi \otimes P \Gamma \Theta_{g}^{-1} \leq 0$ and $2\left(1-\frac{1}{N}\right) I_{N} \otimes\left(\Upsilon_{g}^{\varepsilon}\right)^{2} P \Gamma \Theta_{g}-\eta \Psi \otimes I_{n}-c \Psi \otimes P \Gamma+\Psi \otimes P \Delta \Gamma+c_{2} \Psi B B^{\top} \Psi \otimes P \Gamma \Theta_{h}^{-1}+\Psi \otimes Q+\Psi \otimes R \leq 0$, where $Q=\frac{\zeta}{1-\bar{\tau}_{1}} I_{n}+\nu_{1} I_{n}, R=2 c_{2} \lambda_{\max }(\Psi) \frac{N-1}{N\left(1-\bar{\tau}_{2}\right)}\left(\Upsilon_{h}^{\varepsilon}+\Upsilon_{h}^{\beta}\right)^{2} P \Gamma \Theta_{h}+\nu_{2} I_{n}$, and $\nu_{1}$ and $\nu_{2}$ are positive scalars, we have

$$
\frac{d V(t)}{d t} \leq-\eta \sum_{i=1}^{m} e_{i}(t)^{\top} e_{i}(t)<0
$$

and so $x_{i}(t) \rightarrow s(t)$ and $\dot{c}(t) \rightarrow 0$ (by LaSalle's invariance principle). Therefore $c(t)$ converges to some final coupling strength $c_{0}$.

\section{Numerical Simulation}

In this section, we present some numerical simulation results that validate the theorems of the previous section.

Consider the isolated 2D chaotic delayed neural network

$$
\left[\begin{array}{l}
\dot{s}_{1}(t) \\
\dot{s}_{2}(t)
\end{array}\right]=-\left[\begin{array}{ll}
1 & 0 \\
0 & 1
\end{array}\right]\left[\begin{array}{l}
s_{1}(t) \\
s_{2}(t)
\end{array}\right]+\left[\begin{array}{cc}
2 & -0.1 \\
-5 & 4.5
\end{array}\right]\left[\begin{array}{l}
\tanh \left(s_{1}(t)\right) \\
\tanh \left(s_{2}(t)\right)
\end{array}\right]+\left[\begin{array}{cc}
-1.5 & -0.1 \\
-0.2 & -4
\end{array}\right]\left[\begin{array}{c}
\tanh \left(s_{1}\left(t-\tau_{1}(t)\right)\right) \\
\tanh \left(s_{2}\left(t-\tau_{1}(t)\right)\right)
\end{array}\right]
$$

where $\tau_{1}(t)=1$. Taking $P=\operatorname{diag}\{1,1\}$ and $\Delta=\operatorname{diag}\{5,11.5\}$, we have $\eta=0.15$ and $\zeta=3.25$ so that condition (6) is satisfied.

Now consider a network with 50 nodes with a topological structure that is shown in Figure 1

$\dot{x}_{i}(t)=f\left(t, x_{i}(t), x_{i}\left(t-\tau_{1}(t)\right)\right)+c_{1} \sum_{j=1}^{50} a_{i j} \Gamma g\left(x_{j}(t)\right)+c_{2} \sum_{j=1}^{50} b_{i j} \Gamma h\left(x_{j}\left(t-\tau_{2}(t)\right)\right)-c_{1} \varepsilon_{i} \Gamma\left(x_{i}(t)-s(t)\right), \quad i=1,2, \ldots, 50$,

where $\tau_{2}(t)=0.1 \frac{e^{t}}{1+e^{t}}, g_{j}(x)=25 x+\sin (x)$ and $h_{j}(x)=5 x+\cos (x)$ for $j=1,2, \Gamma=I_{2}$.

Computations then yield $\bar{\tau}_{1}=0$ and $\bar{\tau}_{2}=0.1$ for $i=1,2, \ldots, 50$ and $j=1,2, \Upsilon_{g}^{\beta}=25 I_{2}, \Upsilon_{h}^{\beta}=5 I_{2}, \Upsilon_{g}^{\varepsilon}=I_{2}$, $\Upsilon_{h}^{\varepsilon}=I_{2}$. Let $l=7$ and the control strength $\varepsilon_{i}=100$ for $i=1,2, \ldots, 7$. Then the solutions of inequalities (7) are (by using the Matlab LMI toolbox): $c_{1}=10 c_{2}=0.01, \rho=180.3202 \Theta_{h}=1.9082 I_{2}, \Theta_{g}=41.9134 I_{2}$, $Q=3.251 I_{2}, R=1.497, \nu_{1}=0.001, \nu_{2}=0.001$ and $\mathcal{J}=183.1517$.

The initial conditions for this simulation are $x_{i j}\left(t_{0}\right)=\operatorname{rand}_{i j} \times \cos \left(t_{0}\right)$ for some random integer rand $_{i j}$ in $[0,100]$ for $i=1,2, \ldots, 50, j=1,2$ and $s\left(t_{0}\right)=\left[30 \cos \left(t_{0}\right), 2 \cos \left(t_{0}\right)\right]^{\top}$ for all $t_{0} \in[-1,0]$ and the trajectories of the states are shown in Figure 2. Figure 3 shows the time evolution of the synchronization errors with pinning control. Measuring the quality of the synchronization process by

$$
E(t)=\frac{1}{50} \sum_{i=1}^{50}\left\|x_{i}(t)-s(t)\right\|^{2}
$$

in Figure 4, it is clear that synchronization is achieved.

The above simulations show that large coupling and control strengths $\left(c_{1}\right.$ and $\left.\varepsilon_{i}\right)$ are needed to meet condition (7). Now use the same parameters with $\alpha=0.0001$ to simulate the controlled dynamic network (14) with adaptive coupling strength $c_{1}(t)$. The trajectories of the states are shown in Figure 5 and Figure 6 shows the time evolution of the synchronization errors with pinning control. Figure 7 and Figure 8 show the time evolution of $E(t)$ and $c_{1}(t)$ (note that $c_{1}(t)$ approaches 28.169) for the pinned complex network by adaptive control. It is clear that synchronization is achieved. (Note that the final coupling strength is smaller than its theoretical value.) 


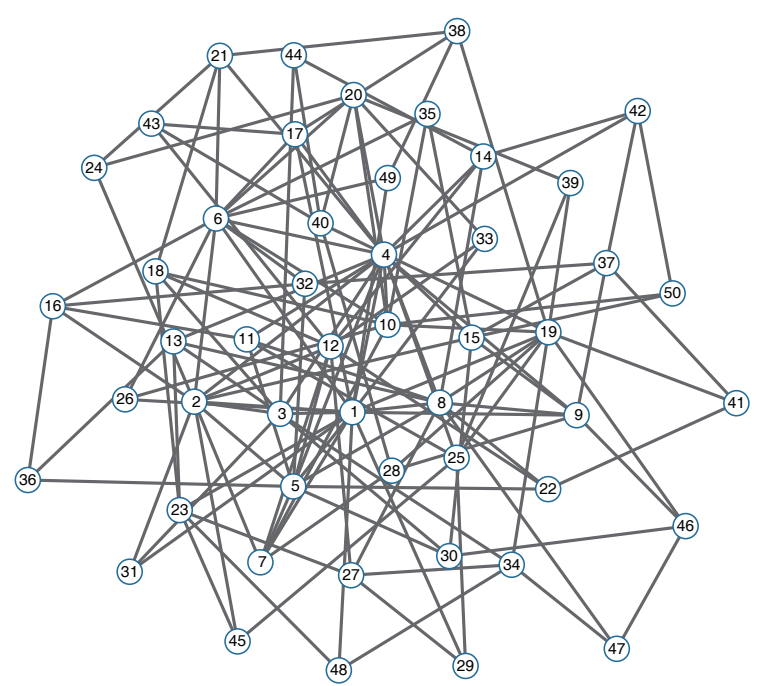

(a) The topological structure of the coupling matrix $A$.

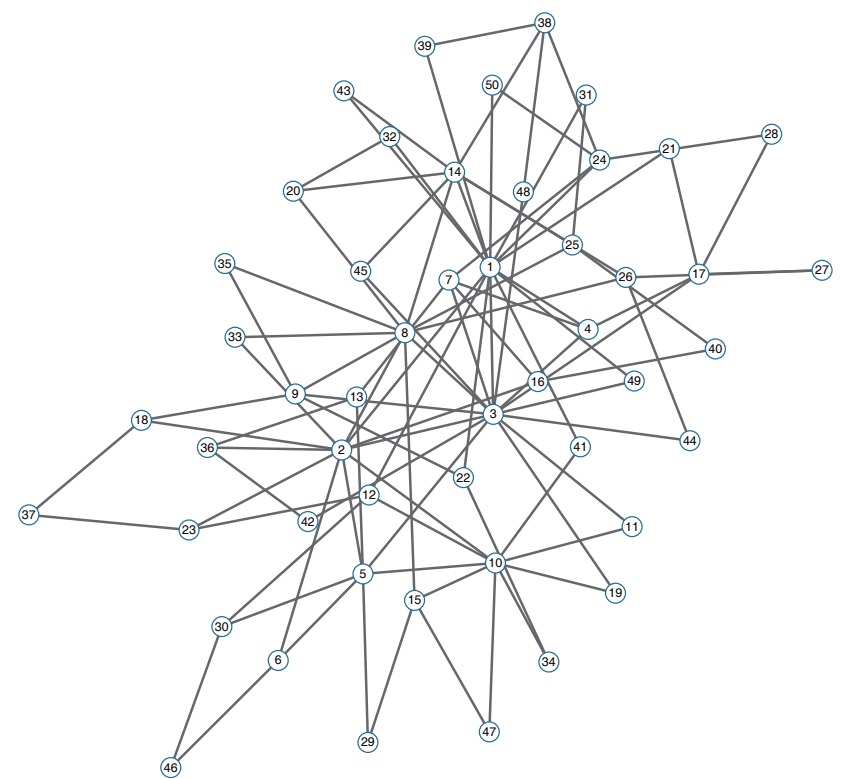

(b) The topological structure of the coupling matrix $B$.

Figure 1: The topological structures of the undirected network with 50 nodes. We assume that $a_{i j}=a_{j i}=1$ if there exists an edge between node $i$ and node $j$ and $a_{i j}=a_{j i}=0$ otherwise in (a); $b_{i j}=b_{j i}=1$ if there exists an edge between node $i$ and node $j$ and $b_{i j}=b_{j i}=0$ otherwise in (b).

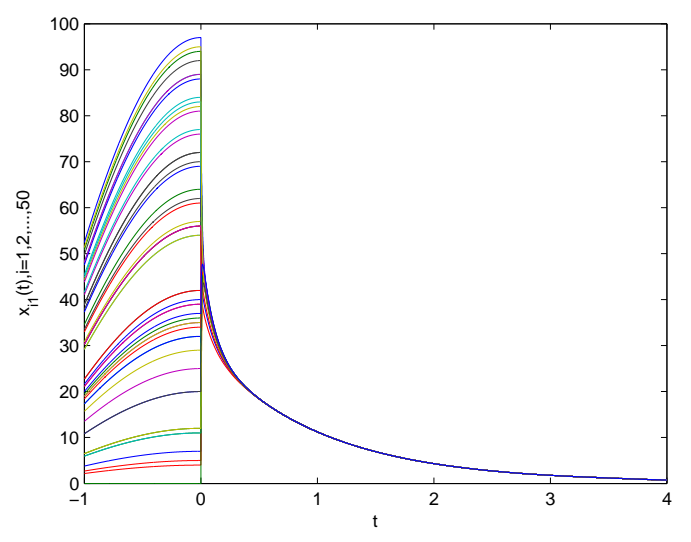

(a)

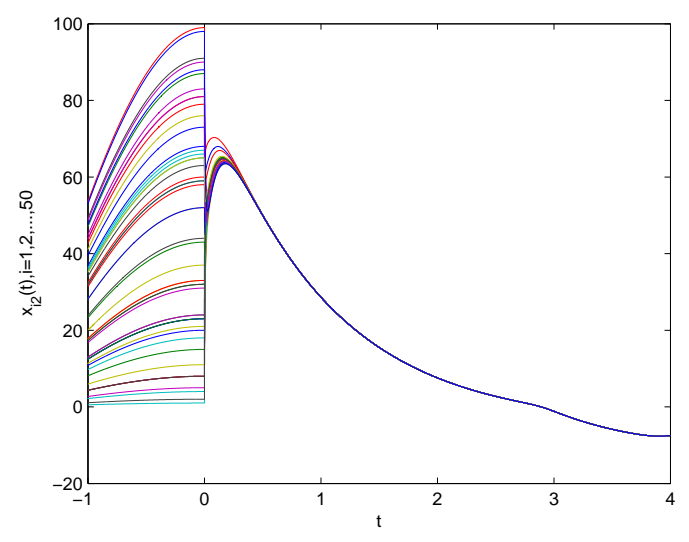

(b)

Figure 2: The trajectories of the state variables of $x_{i 1}(\mathrm{a})$ and $x_{i 2}(\mathrm{~b})$ for $i=1,2, \ldots, 50$ in system (15) using pinning control. 


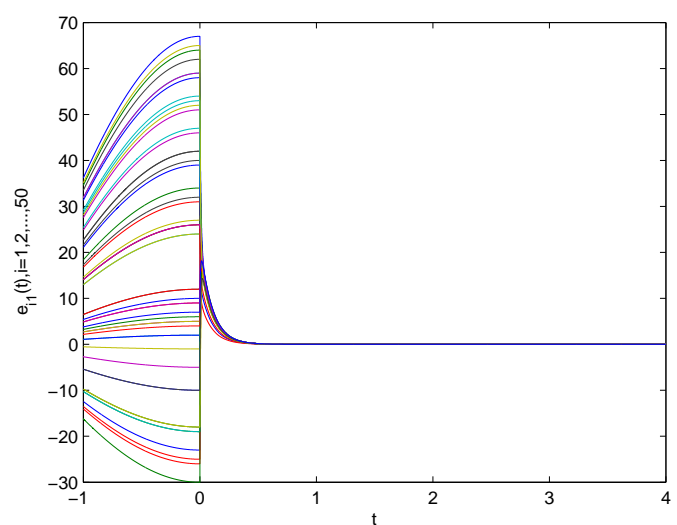

(a)

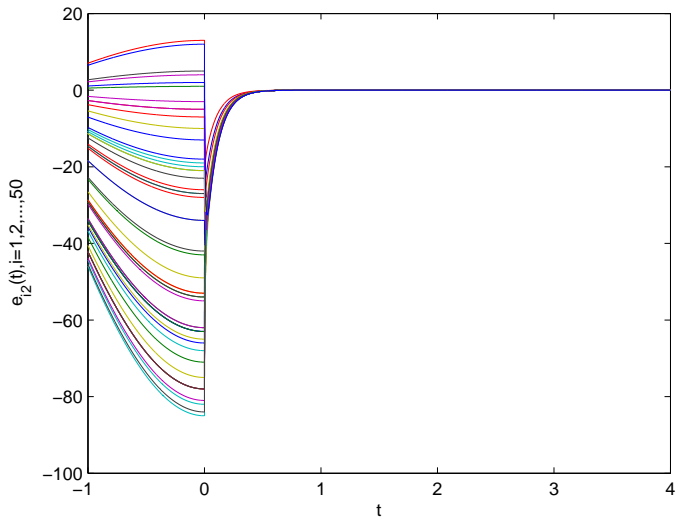

(b)

Figure 3: The time-evolution of $e_{i 1}$ (a) and $e_{i 2}$ (b) for $i=1,2, \ldots, 50$ in system (15) using pinning control.

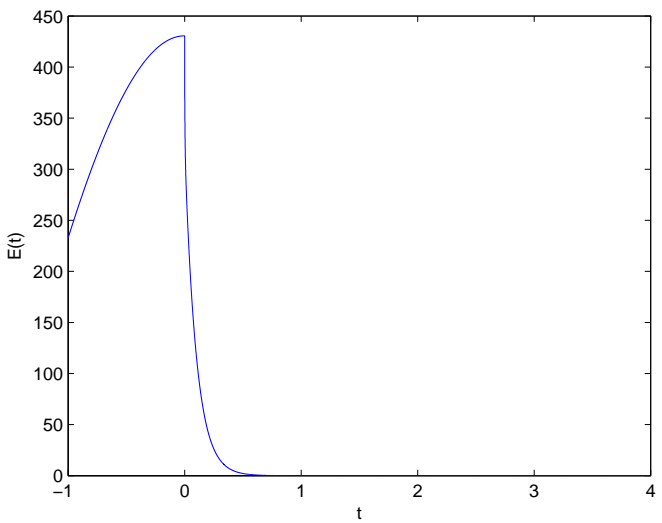

Figure 4: The synchronization process in system (15) using pinning control.

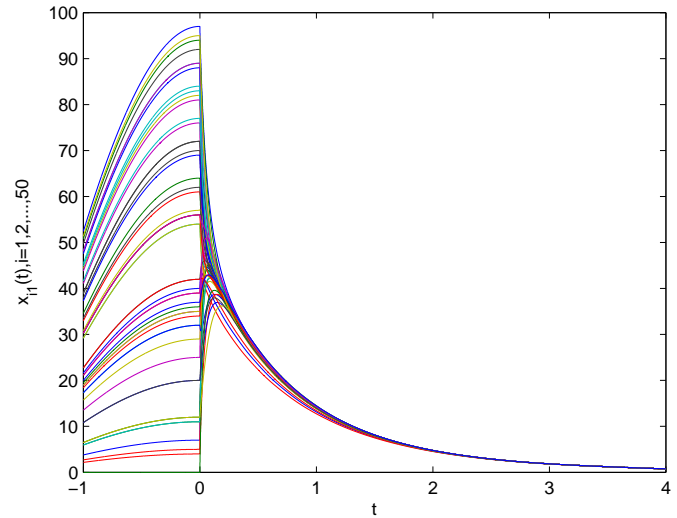

(a)

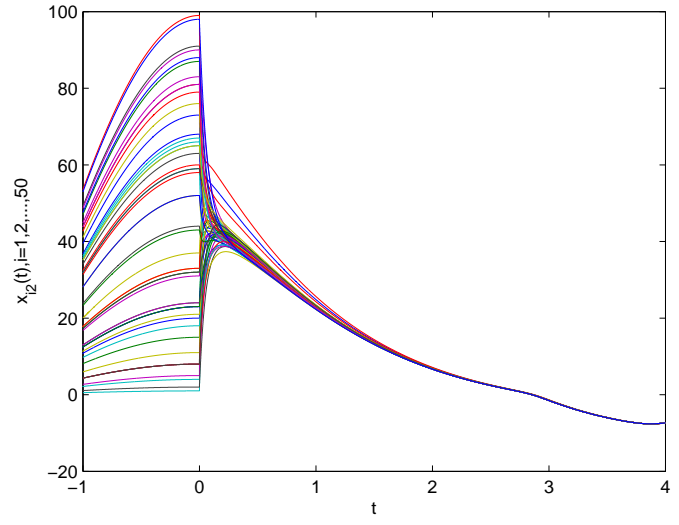

(b)

Figure 5: The trajectories of the state variables of $x_{i 1}(\mathrm{a})$ and $x_{i 2}(\mathrm{~b})$ for $i=1,2, \ldots, 50$ in system (15) using adaptive and pinning control.

10 


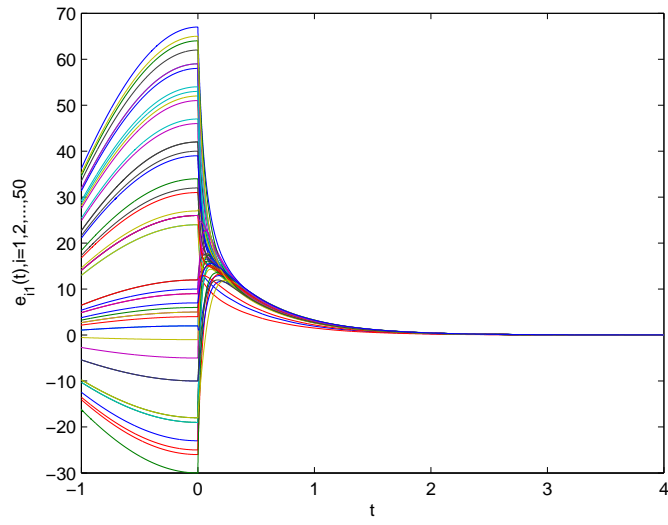

(a)

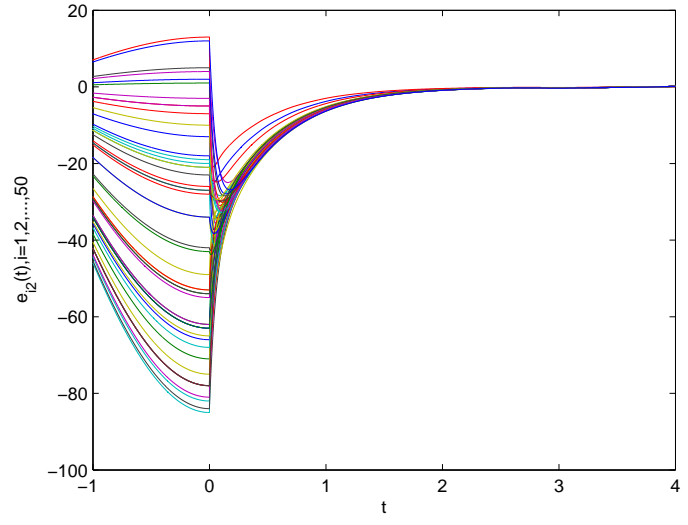

(b)

Figure 6: The time-evolution of $e_{i 1}$ (a) and $e_{i 2}$ (b) for $i=1,2, \ldots, 50$ in system (15) using adaptive and pinning control.

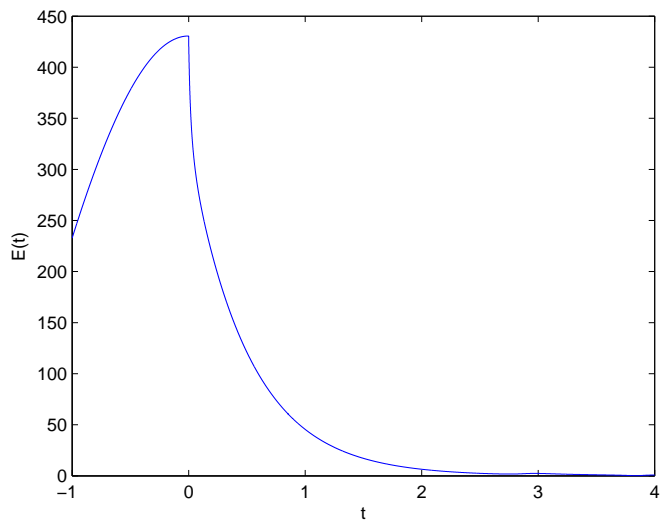

Figure 7: The synchronization process of system (15) using adaptive and pinning control.

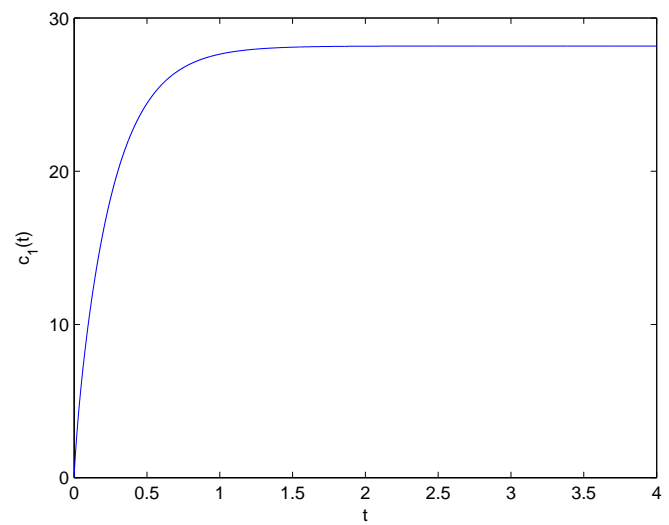

Figure 8: The adaptive coupling strength $c_{1}(t)$ of the pinning-controlled complex network 


\section{Conclusion}

In this paper, we investigated the synchronization problem for complex networks with non-delayed and time-varying delayed couplings. Specifically, we achieved global synchronization by applying a pinning control scheme to a small fraction of the nodes using the M-matrix strategies. Noting the big difference in the coupling strength magnitudes between theoretical and real-world systems, we also rigorously proved an adaptive feedback control technique that can be used to synchronize any real-world network. Finally, we considered some numerical examples that illustrate the theoretical analysis.

\section{Acknowledgment}

This work was supported by the National Science Foundation of China under Grant No.61273220, 61373087, 61472257 and 61401283 . The authors are grateful to the reviewers and editors for their valuable comments and suggestions to improve the presentation of this paper.

\section{References}

[1] A.-L. Barabási, R. Albert, Emergence of scaling in random networks, Science 286 (5439) (1999) 509-512. doi:10.1126/ science.286.5439.509.

[2] A. Barrat, M. Weigt, On the properties of small-world network models, The European Physical Journal B-Condensed Matter and Complex Systems 13 (3) (2000) 547-560.

[3] A.-L. Barabási, Z. N. Oltvai, Network biology: understanding the cell's functional organization, Nature Reviews Genetics 5 (2) (2004) 101-113.

[4] A.-L. Barabási, The network takeover, Nature Physics 8 (1) (2011) 14-16.

[5] D. Helbing, Globally networked risks and how to respond, Nature 497 (7447) (2013) 51-59.

[6] M. Boguñá, D. Krioukov, Navigating ultrasmall worlds in ultrashort time, Physical Review Letters 102 (2009) 058701. doi: 10.1103/prl.102.058701. URL http://link.aps.org/doi/10.1103/prl.102.058701

[7] R. Lu, W. Yu, J. Lü, A. Xue, Synchronization on complex networks of networks, IEEE Transactions on Neural Networks and Learning Systems 25 (11) (2014) 2110-2118. doi:10.1109/TNNLS.2014.2305443.

[8] S. Boccaletti, J. Kurths, G. Osipov, D. L. Valladares, C. Zhou, The synchronization of chaotic systems, Physics Reports 366 (1-2) (2002) 1-101. doi:10.1016/S0370-1573(02)00137-0.

[9] X. F. Wang, G. Chen, Synchronization in scale-free dynamical networks: robustness and fragility, IEEE Transactions on Circuits and Systems I: Fundamental Theory and Applications 49 (1) (2002) 54 -62. doi:10.1109/81.974874.

[10] F. Dörfler, F. Bullo, Synchronization in complex networks of phase oscillators: A survey, Automatica 50 (6) (2014) 1539 1564. doi:http://dx.doi.org/10.1016/j.automatica.2014.04.012. URL http://www.sciencedirect.com/science/article/pii/S0005109814001423

[11] R. Tönjes, B. Blasius, Perturbation analysis of complete synchronization in networks of phase oscillators, Physical Review E 80 (2009) 026202. doi:10.1103/PhysRevE.80.026202. URL http://link.aps.org/doi/10.1103/PhysRevE.80.026202

[12] J. Lunze, Complete synchronization of kuramoto oscillators, Journal of Physics A: Mathematical and Theoretical 44 (42) (2011) 425102 .

[13] W. Wu, W. Zhou, T. Chen, Cluster synchronization of linearly coupled complex networks under pinning control, IEEE Transactions on Circuits and Systems I: Regular Papers 56 (4) (2009) 829 -839. doi:10.1109/TCSI.2008.2003373.

[14] M. G. Rosenblum, A. S. Pikovsky, J. Kurths, Phase synchronization of chaotic oscillators, Physical Review Letters 76 (1996) 1804-1807, phase Synchronization. doi:10.1103/PhysRevLett.76.1804.

URL http://link.aps.org/doi/10.1103/PhysRevLett.76.1804

[15] J. Lu, X. Wu, X. Han, J. Lü, Adaptive feedback synchronization of a unified chaotic system, Physics Letters A 329 (4) (2004) $327-333$.

[16] H. Zhang, M. Zhao, Z. Wang, Z. Wu, Adaptive synchronization of an uncertain coupling complex network with time-delay, Nonlinear Dynamics 77 (3) (2014) 643-653. doi:\{10.1007/s11071-014-1327-z\}.

[17] J. Wang, J. Feng, C. Xu, Y. Zhao, Exponential synchronization of stochastic perturbed complex networks with time-varying delays via periodically intermittent pinning, Communications in Nonlinear Science and Numerical Simulation 18 (11) (2013) 3146 - 3157. doi:http://dx.doi.org/10.1016/j.cnsns.2013.03.021. URL http://www.sciencedirect.com/science/article/pii/S1007570413001676

[18] G. Zhang, Z. Liu, Z. Ma, Synchronization of complex dynamical networks via impulsive control, Chaos: An Interdisciplinary Journal of Nonlinear Science 17 (4) (2007) 043126-043126.

[19] T. Chen, X. Liu, W. Lu, Pinning complex networks by a single controller, IEEE Transactions on Circuits and Systems I: Regular Papers, 54 (6) (2007) 1317-1326. doi:10.1109/TCSI.2007.895383.

[20] W. Yu, G. Chen, J. Lü, On pinning synchronization of complex dynamical networks, Automatica 45 (2) (2009) $429-435$.

[21] J. Zhao, J. Lu, X. Wu, Pinning control of general complex dynamical networks with optimization, SCIENCE CHINA Information Sciences 53 (2010) 813-822. URL http://dx.doi.org/10.1007/s11432-010-0039-3

[22] R. Cheng, M. Peng, W. Yu, Pinning synchronization of delayed complex dynamical networks with nonlinear coupling, Physica A: Statistical Mechanics and its Applications 413 (2014) 426-431. doi:http://dx.doi.org/10.1016/j.physa.2014.06.034.

[23] W. Yu, G. Chen, J. Lu, J. Kurths, Synchronization via pinning control on general complex networks, SIAM Journal on Control and Optimization 51 (2) (2013) 1395-1416. doi:\{10.1137/100781699\}.

[24] Q. Song, J. Cao, On pinning synchronization of directed and undirected complex dynamical networks, IEEE Transactions on Circuits and Systems I: Regular Papers 57 (3) (2010) 672-680. doi:10.1109/TCSI.2009.2024971. 
[25] J. Wang, J. Feng, C. Xu, Y. Zhao, Cluster synchronization of nonlinearly-coupled complex networks with nonidentical nodes and asymmetrical coupling matrix, Nonlinear Dynamics 67 (2) (2012) 1635-1646.

[26] H. Su, X. Wang, Pinning control of complex networked systems: Synchronization, consensus and flocking of networked systems via pinning, Springer, Berlin, 2013

[27] Q. Song, F. Liu, J. Cao, W. Yu, Pinning-controllability analysis of complex networks: An m-matrix approach, IEEE Transactions on Circuits and Systems I: Regular Papers 59 (11) (2012) 2692-2701. doi:10.1109/TCSI.2012.2190573.

[28] Q. Song, F. Liu, J. Cao, W. Yu, M-matrix strategies for pinning-controlled leader-following consensus in multiagent systems with nonlinear dynamics, IEEE Transactions on Cybernetics 43 (6) (2013) 1688-1697. doi:10.1109/TSMCB.2012.2227723.

[29] G. Wen, Z. Duan, G. Chen, W. Yu, Consensus tracking of multi-agent systems with lipschitz-type node dynamics and switching topologies, Circuits and Systems I: Regular Papers, IEEE Transactions on 61 (2) (2014) 499-511. doi:10.1109/ TCSI. 2013.2268091

[30] R. A. Horn, C. R. Johnson, Topics in Matrix Analysis, Cambrigdge University Press, New York, 1994.

[31] C. Li, W. Yu, T. Huang, Impulsive synchronization schemes of stochastic complex networks with switching topology: Average time approach, Neural Networks 54 (0) (2014) $85-94$. 